\title{
Successful closure using endoscopic ligation with O-ring and over- the-scope clip in gastric iatrogenic perforation
}

\author{
Tatsuo Yachidaa,b, Hideki Kobaraa ${ }^{a}$, Noriko Nishiyama ${ }^{a}$, Tsutomu Masakia
}

Kagawa University, Kagawa, Japan

Closure using a conventional hemoclip is limited to managing immediate large perforations during endoscopic submucosal dissection (ESD). Innovative closure techniques using an overthe-scope clip (OTSC) with endoloop and others have been developed [1]; however, this requires familiarity with a singlechannel scope to make sure that full-thickness closure is achieved without delayed leakage. For approximating large perforations with the aid of a Twin Grasper ([TG], Ovesco Endoscopy), using OTSC is challenging because of the difficulty in maneuvering the TG and the risk of insufflation leakage [2]. The technique of endoscopic ligation with O-ring closure (E-LOC) that we have developed [3] seems advantageous for overcoming this problem. Here we describe the successful closure of a large iatrogenic perforation using E-LOC followed by OTSC.

A 63-year-old man presented with early gastric cancer located in the fornix. A large perforation $(20 \mathrm{~mm})$ occurred during ESD (Fig. 1A). After en bloc resection with snaring and unsuccessful closure using hemoclips (Fig. 1B), E-LOC followed by OTSC was attempted once written informed consent had been obtained. First, a 2-cm diameter 3-0 surgical nylon loop was positioned around the defect and was anchored using two hemoclips on both edges (Fig. 1C). Then, the grasping forceps that grasped the loop was pulled into the endoscopic variceal ligation cap, so that the 2 deployed hemoclips were captured within the cap (Fig. 1D). An O-ring was fired around these hemoclips to approximate the large defect (Fig. 2A). Finally, 2 OTSCs (gc type, $10 \mathrm{~mm}$ ) were easily deployed for complete full-thickness closure (Fig. 2B,C). One month later, the closure remained intact (Fig. 2D).

This procedure may be a reliable option for managing large perforations.

\section{Acknowledgment}

The authors wish to thank Dr. Tomohiro Ohgi, HITO Medical Center, for his assistance in this study.

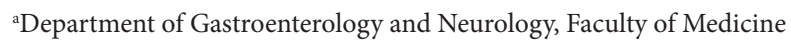
(Tatsuo Yachida, Hideki Kobara, Noriko Nishiyama, Tsutomu Masaki); ${ }^{b}$ Department of General Internal Medicine, Faculty of Medicine (Tatsuo Yachida), Kagawa University, Kagawa, Japan

Conflict of Interest: None

Correspondence to: Tatsuo Yachida, M.D, PhD, Department of General Internal Medicine, Faculty of Medicine, Kagawa University, 1750-1 Ikenobe, Miki, Kita, Kagawa 761-0793, Japan,

e-mail: tyachida@med.kagawa-u.ac.jp

Received 11 December 2020; accepted 30 December 2020; published online 26 February 2021

DOI: https://doi.org/10.20524/aog.2021.0611

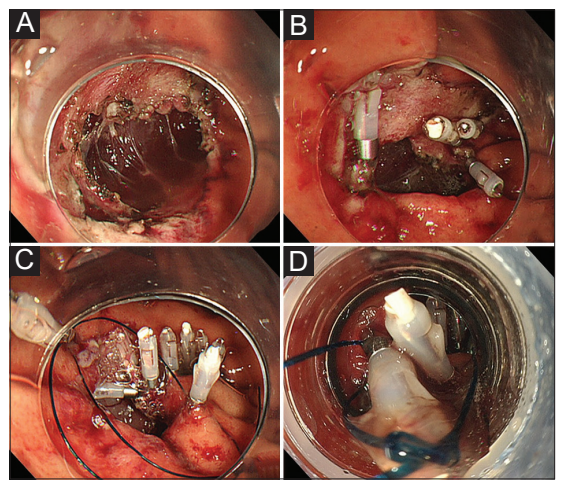

Figure 1 (A) A large perforation (20-mm) during endoscopic submucosal dissection. (B) Unsuccessful closure using hemoclips. (C) A 2-cm diameter 3-0 surgical nylon loop is positioned around the defect, and the loop is anchored using 2 hemoclips on both edges of the defect. (D) The grasping forceps (FG-47L-1; Olympus, Tokyo, Japan) that grasped the loop is pulled into the cap of the endoscopic variceal ligation device (MD-48720U; Sumius, Tokyo, Japan) and the 2 deployed hemoclips are captured within the cap

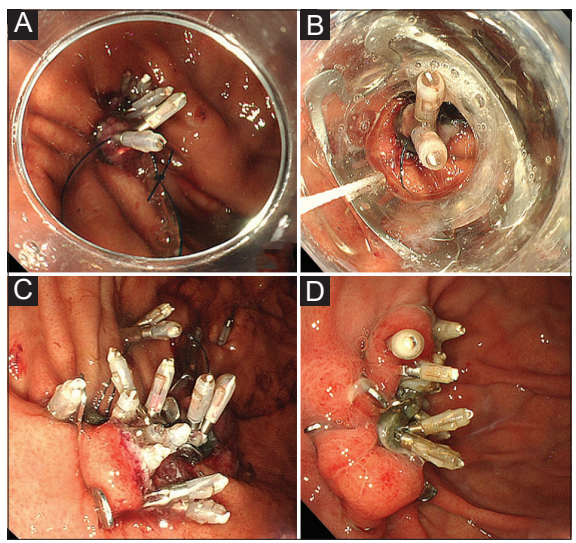

Figure 2 (A) An O-ring is fired around these hemoclips, succeeding in approximating the large defect. (B) The O-ring and hemoclips are inserted into the cap with the over-the-scope clip (OTSC) (Ovesco Endoscopy AG, Tübingen, Germany) attached. (C) Two OTSCs are deployed, covering the prepared hemoclips for complete full-thickness closure. (D) One month later, the closure is intact

\section{References}

1. Tashima T, Nonaka K, Ryozawa S, Tanisaka Y. Endoscopic pursestring suturing with an over-the-scope clip for closure of a large mucosal defect after gastric ESD. Dig Liver Dis 2018;50:1247.

2. Kobara H, Mori H, Nishiyama N, et al. Over-the-scope clip system: a review of 1517 cases over 9 years. J Gastroenterol Hepatol 2019;34:22-30.

3. Nishiyama N, Kobara H, Kobayashi N, et al. Novel endoscopic ligation with O-ring closure involving muscle layer of a gastric artificial defect. Endoscopy 2020;52:E413-E414. 